\title{
SOME OBSERVATIONS ON THE DEVELOPMENT OF HYPOKALIEMIA DURING THERAPY OF DIABETIC ACIDOSIS IN JUVENILE AND YOUNG ADULT SUBJECTS
}

\author{
By L. GREENMAN, F. M. MATEER, R. C. GOW, J. H. PETERS, ANd T. S. DANOWSKI \\ (From the Department of Rescarch Medicine, the Children's and Presbyterian Hospitals, and \\ the Renziehausen Foundation, University of Pittsburgh School of Medicine, Pittsburgh)
}

(Received for publication October 18, 1948)

It is known that hypopotassemia may appear during therapy of diabetic acidosis or coma, at times associated with muscular paralysis (1-9). This is not, however, an invariable occurrence, nor have the origins of such decreases in the serum and interstitial fluid potassium concentrations been clearly defined. It seems probable that one or more mechanisms are responsible. These include the possibilities that hypokaliemia ${ }^{1}$ develops as a result of a continued loss of potassium in urine, that it is a manifestation of dilution of body fluids by potassium-free solutions administered during therapy, and, finally, that it results from movements of potassium into body cells. The studies herein reported evaluate the role of each of these factors in a limited number of patients, chiefly pre-adult, in whom intensive study using the balance method was possible.

\section{MATERIALS AND METHODS}

Nine patients have been studied during a total of 11 admissions for the treatment of diabetic acidosis. Two of the subjects were young adults; the remainder were children seven to 16 years of age. Over-breathing was definitely present in each case; the serum carbon dioxide content ranged from 2.6 to 10.4 m.eq. per liter on admission. All patients were conscious, though difficult to arouse. Definite vascular collapse was present in only one patient, R. J., requiring three transfusions. Anuria did not develop in any of the subjects. Insulin was administered subcutaneously to all patients, although J. K. and $\mathrm{T}$. W. also received large intravenous injections initially. Data on the retention of potassium salts administered in large amounts during convalescence to eight of the cases have been presented in a previous communication (9). The studies herein reported are based on observations from the time of admission and continued until convalescence was well established. During these inter-

1 This term has been taken to refer solely to diminished concentrations of potassium in blood serum or plasma and the contiguous interstitial fluid, and does not necessarily indicate any decrease in the total amount of potassium in these fluids. vals the patients received insulin, glucose, saline, and only little, if any, potassium.

The experimental procedure employed and the methods of calculation have been previously described in detail (9-12). Briefly they may be summarized as follows: the chloride space, corrected for the external balance of this ion and for changes in the chloride concentration in serum, has been used as an index of alterations in the volume of extracellular fluid. The intake and the urinary excretion of sodium, potassium, chloride, glucose, and nitrogen were measured during intervals marked at the start and finish by determinations of the values of these various components in serum. The retention or loss of these cations and of nitrogen has been apportioned between the extracellular fluid and the cells. In the case of nitrogen, suitable corrections for changes in the concentration of the whole blood nonprotein nitrogen were made. The values for the cell balances of potassium represent, therefore, changes in excess of those which might be expected with the anabolism or catabolism of cell protein, employing the usual ratio of 1 gram nitrogen : 2.38 m.eq. of potassium.

It must be emphasized that the experimental procedure and the methods of calculation employed yield only the over-all balance of any particular component studied. Thus it should be clear that if the over-all loss of potassium from cells during any individual period should prove to be 40 m.eq., the possibility cannot be excluded, for example, that muscle cells actually lost $70 \mathrm{~m}$.eq. but that this was reduced to $40 \mathrm{~m}$.eq. by a movement of $30 \mathrm{~m} . e q$. into the liver with glycogen.

\section{RESULTS}

From Table $I$ it is evident that during treatment the serum potassium declined progressively to abnormally low levels in seven of the 11 cases. In two instances in which this did not occur, patients L. B. and M. S., hypopotassemia was already present on admission. In the former the potassium level remained low, while in the latter a rise was observed. In the two other patients, J. K. (a) and W. S., potassium decreased during treatment, but remained within normal limits. ${ }^{2}$

${ }^{2}$ All patients, save $R$. J., had received insulin, protamine or regular, within the 24 hours preceding admission. 
TABLE I

Blood and serum analyses prior to and during therapy of diabetic acidosis or coma

\begin{tabular}{|c|c|c|c|c|c|c|c|c|c|}
\hline \multirow{2}{*}{$\begin{array}{c}\text { Patient } \\
\text { (Age-Sex) }\end{array}$} & \multirow{2}{*}{$\begin{array}{l}\text { Time } \\
\text { from } \\
\text { start }\end{array}$} & \multirow{2}{*}{$\begin{array}{l}\text { Body } \\
\text { Wgt. }\end{array}$} & \multicolumn{2}{|c|}{ Blood } & \multicolumn{5}{|c|}{ Serum } \\
\hline & & & NPN & Sugar & $\mathrm{HCO}_{3}$ & $\mathrm{Cl}$ & $\mathrm{Na}$ & $\mathrm{K}$ & $\mathrm{H}_{2} \mathrm{O}$ \\
\hline $\begin{array}{l}\text { L. B. } \\
(12 \mathrm{M})\end{array}$ & $\begin{array}{r}\text { hours } \\
0 \\
5.5 \\
18.3 \\
19.5\end{array}$ & $\begin{array}{c}\text { kgm. } \\
30.5 \\
30.9 \\
31.3 \\
-\end{array}$ & $\begin{array}{c}m g m . \% \\
27 \\
25 \\
-\end{array}$ & $\begin{array}{c}\text { mgm. \% } \\
232 \\
176 \\
70 \\
-\end{array}$ & $\begin{array}{c}\text { m.eq. per } \\
\text { liter } \\
6.1 \\
13.0 \\
17.2 \\
-\end{array}$ & $\begin{array}{c}\text { m.eq. per } \\
\text { liter } \\
97.5 \\
105.8 \\
98.9 \\
102.4\end{array}$ & $\begin{array}{c}\text { m.eq. per } \\
\text { liter } \\
120.3 \\
128.5 \\
138.9 \\
141.4\end{array}$ & $\begin{array}{c}\text { m.eq. per } \\
\text { liter } \\
3.0 \\
- \\
3.0 \\
3.0\end{array}$ & $\begin{array}{c}\text { gms. per } \\
\text { liter } \\
\text { - }^{*} \\
929 \\
932 \\
930\end{array}$ \\
\hline $\begin{array}{c}\text { D. C. (a) } \\
(16 \mathrm{~F})\end{array}$ & $\begin{array}{r}0 \\
5.0 \\
10.0 \\
22.8\end{array}$ & $\begin{array}{c}52.7 \\
- \\
53.4\end{array}$ & $\begin{array}{l}65 \\
57 \\
41 \\
28\end{array}$ & $\begin{array}{l}582 \\
327 \\
154 \\
171\end{array}$ & $\begin{array}{r}4.8 \\
5.2 \\
9.8 \\
15.1\end{array}$ & $\begin{array}{r}89.9 \\
98.3 \\
111.5 \\
111.5\end{array}$ & $\begin{array}{l}122.7 \\
128.9 \\
144.1 \\
155.9\end{array}$ & $\begin{array}{l}6.5 \\
5.1 \\
3.5 \\
3.0\end{array}$ & $\begin{array}{l}899 \\
911 \\
930 \\
941\end{array}$ \\
\hline $\begin{array}{c}\text { D. C. }(\mathrm{b}) \\
(16 \mathrm{~F})\end{array}$ & $\begin{array}{r}0 \\
5.5 \\
19.2\end{array}$ & $\frac{50.0}{53.9}$ & $\begin{array}{l}67 \\
53 \\
28\end{array}$ & $\begin{array}{l}786 \\
456 \\
163\end{array}$ & $\begin{array}{r}6.8 \\
8.5 \\
15.4\end{array}$ & $\begin{array}{r}91.4 \\
111.2 \\
117.1\end{array}$ & $\begin{array}{l}125.3 \\
138.6 \\
145.6\end{array}$ & $\begin{array}{l}6.7 \\
4.8 \\
3.3\end{array}$ & $\begin{array}{l}907 \\
921 \\
936\end{array}$ \\
\hline $\begin{array}{l}\text { L. D. } \\
(15 \mathrm{M})\end{array}$ & $\begin{array}{r}0 \\
5.0 \\
8.0 \\
15.0 \\
23.0\end{array}$ & $\begin{array}{l}38.7 \\
- \\
- \\
38.3\end{array}$ & $\begin{array}{l}33 \\
25 \\
22 \\
23 \\
24\end{array}$ & $\begin{array}{r}1189 \\
240 \\
275 \\
73 \\
51\end{array}$ & $\begin{array}{r}4.1 \\
5.7 \\
9.9 \\
12.0 \\
11.2\end{array}$ & $\begin{array}{r}96.5 \\
110.4 \\
111.1 \\
111.4 \\
109.3\end{array}$ & $\begin{array}{l}124.2 \\
145.5 \\
147.3 \\
135.7 \\
143.9\end{array}$ & $\begin{array}{l}4.0 \\
2.6 \\
2.9 \\
1.8 \\
1.8\end{array}$ & $\begin{array}{l}924 \\
934 \\
941 \\
940 \\
943\end{array}$ \\
\hline $\begin{array}{c}\text { R. J. } \\
(14 \mathrm{M})\end{array}$ & $\begin{array}{r}0 \\
4.0 \\
6.8 \\
10.8 \\
16.8 \\
19.2\end{array}$ & $\begin{array}{c}34.9 \\
- \\
- \\
- \\
-\end{array}$ & $\begin{array}{r}103 \\
100 \\
105 \\
89 \\
79 \\
79\end{array}$ & $\begin{array}{r}1395 \\
1165 \\
895 \\
736 \\
304 \\
230\end{array}$ & $\begin{array}{l}10.4 \\
17.5 \\
18.1 \\
19.6 \\
19.5 \\
20.6\end{array}$ & $\begin{array}{l}101.9 \\
115.0 \\
122.7 \\
134.6 \\
145.2 \\
137.3\end{array}$ & $\begin{array}{l}142.1 \\
152.8 \\
156.6 \\
162.2 \\
165.1 \\
161.6\end{array}$ & $\begin{array}{l}3.7 \\
3.2 \\
3.2 \\
2.9 \\
2.8 \\
2.0\end{array}$ & $\begin{array}{l}912 \\
916 \\
919 \\
930 \\
936 \\
933\end{array}$ \\
\hline $\begin{array}{c}\mathrm{J} . \mathrm{K} .(\mathrm{a}) \\
(18 \mathrm{~F})\end{array}$ & $\begin{array}{r}0 \\
20.0\end{array}$ & $\begin{array}{l}50.0 \\
53.9\end{array}$ & $\begin{array}{l}32 \\
21\end{array}$ & $\begin{array}{l}600 \\
145\end{array}$ & $\begin{array}{r}8.1 \\
19.0\end{array}$ & $\begin{array}{l}94.1 \\
97.8\end{array}$ & $\begin{array}{l}135.5 \\
140.5\end{array}$ & $\begin{array}{l}5.4 \\
5.0\end{array}$ & $\begin{array}{l}916 \\
934\end{array}$ \\
\hline $\begin{array}{c}\mathrm{J} \cdot \mathrm{K} .(\mathrm{b}) \\
(18 \mathrm{~F})\end{array}$ & $\begin{array}{r}0 \\
8.8 \\
20.3\end{array}$ & $\begin{array}{l}50.7 \\
52.6 \\
52.8\end{array}$ & $\begin{array}{l}29 \\
24 \\
21\end{array}$ & $\begin{array}{r}372 \\
266 \\
71 \\
\end{array}$ & $\begin{array}{r}2.6 \\
7.9 \\
13.9\end{array}$ & $\begin{array}{l}103.9 \\
120.3 \\
112.9\end{array}$ & $\begin{array}{l}116.3 \\
140.3 \\
129.9\end{array}$ & $\begin{array}{l}3.4 \\
2.9 \\
2.9\end{array}$ & $\begin{array}{l}910 \\
932 \\
944\end{array}$ \\
\hline $\begin{array}{l}\text { W.S. } \\
(14 \mathrm{M})\end{array}$ & $\begin{array}{r}0 \\
4.5 \\
12.2\end{array}$ & $\begin{array}{c}36.2 \\
- \\
-\end{array}$ & $\begin{array}{l}39 \\
35 \\
21\end{array}$ & $\begin{array}{r}470 \\
270 \\
70\end{array}$ & $\begin{array}{r}6.7 \\
7.8 \\
14.7\end{array}$ & $\begin{array}{r}96.3 \\
100.5 \\
114.9\end{array}$ & $\begin{array}{l}129.9 \\
139.9 \\
149.6\end{array}$ & $\begin{array}{l}5.5 \\
5.1 \\
4.1\end{array}$ & $\begin{array}{l}912 \\
931 \\
943\end{array}$ \\
\hline $\begin{array}{l}\text { M.S. } \\
(15 F)\end{array}$ & $\begin{array}{r}0 \\
3.8 \\
17.0\end{array}$ & $\begin{array}{l}41.5_{\ddagger}^{+} \\
-\end{array}$ & $\frac{65}{36}$ & $\begin{array}{l}667 \\
266 \\
104\end{array}$ & $\begin{array}{r}9.3 \\
13.5 \\
17.4\end{array}$ & $\begin{array}{r}77.6 \\
93.1 \\
107.7\end{array}$ & $\begin{array}{l}118.6 \\
128.0 \\
144.6\end{array}$ & $\begin{array}{l}3.4 \\
3.8 \\
3.9\end{array}$ & $\begin{array}{l}897 \\
927 \\
943\end{array}$ \\
\hline $\begin{array}{l}\text { T. W. } \\
(26 \mathrm{~F})\end{array}$ & $\begin{array}{r}0 \\
9.0 \\
21.0 \\
25.3\end{array}$ & $\begin{array}{l}55.0_{+}^{+} \\
- \\
-\end{array}$ & $\begin{array}{l}-\dagger \\
\overline{40}\end{array}$ & $\begin{array}{l}572 \\
364 \\
284 \\
112\end{array}$ & $\begin{array}{r}5.5 \\
\overline{8.9} \\
11.8\end{array}$ & $\begin{array}{l}101.9 \\
112.6 \\
123.9 \\
127.8\end{array}$ & $\begin{array}{l}142.3 \\
143.7 \\
154.3 \\
157.6\end{array}$ & $\begin{array}{l}4.0 \\
3.8 \\
3.1 \\
2.8\end{array}$ & $\begin{array}{l}{ }^{*} \\
934 \\
940 \\
951\end{array}$ \\
\hline$\underset{(7 \mathrm{M})}{\mathrm{J} . \mathrm{Ko}}$ & $\begin{array}{r}0 \\
6.5 \\
23.0 \\
71.0\end{array}$ & $\frac{21.3}{-}$ & $\begin{array}{l}36 \\
24 \\
- \\
-\end{array}$ & $\begin{array}{l}429 \\
348 \\
198 \\
298\end{array}$ & $\begin{array}{r}4.1 \\
10.6 \\
14.1 \\
23.6\end{array}$ & $\begin{array}{r}96.8 \\
113.9 \\
107.6 \\
92.7\end{array}$ & $\begin{array}{l}123.3 \\
123.3 \\
135.5 \\
135.1\end{array}$ & $\begin{array}{l}5.2 \\
2.7 \\
2.2 \\
2.1\end{array}$ & $\begin{array}{l}926 \\
947 \\
939 \\
946\end{array}$ \\
\hline
\end{tabular}

* Average value of the other cases used in calculating concentrations in extracellular water.

$\dagger$ NPN of 60 on admission assumed for calculation of cell $\mathrm{N}$ balances. solids.

$\ddagger$ Represents weight obtained by calculation, using subsequent weight corrected for intake and losses of water and

Throughout these early hours of therapy each patient continued to excrete urine which contained measurable amounts of potassium (Table II). In no instance did the urinary excretion of this ion decline to zero. These findings are in keeping with those of Atchley et al., whose subjects also continued to excrete potassium during the period of treatment (13). The magnitude of this loss 
in the study cited was less than in the pretreatment period, but in no instance was it entirely cancelled.

Furthermore, comparison of the external and the extracellular balances of potassium, as tabulated in Table III, indicates clearly that, with but one significant exception, the cells were losing potassium as serum levels declined. Only one patient, T. W., had received enough potassium in the intake to produce a positive balance of cell potassium. It is to be noted that these negative balances of cell potassium cannot be attributed solely to the breakdown of protein. This is apparent from the net balances of cell $\mathrm{N}$ corrected for changes in the NPN, as recorded in the final section of Table III. Using the $2.38: 1$ cell $\mathrm{K}: \mathrm{N}$ ratio it may be seen that in no instance was the negative cell $\mathrm{N}$ balance of sufficient magnitude to account for the entire loss of cell potassium. Neither were the negative balances of cell potassium cancelled when cell $\mathrm{N}$ balances became positive. It would appear therefore from the above data that, based on the methods of calculation described earlier, potassium continued to pour out

TABLE II

Intake and output data

\begin{tabular}{|c|c|c|c|c|c|c|c|c|c|c|c|c|}
\hline \multirow{2}{*}{ Pt. } & \multirow{2}{*}{$\begin{array}{l}\text { Time } \\
\text { from } \\
\text { start }\end{array}$} & \multicolumn{5}{|c|}{ Intake } & \multicolumn{6}{|c|}{ Urine output } \\
\hline & & Insulin & $\mathrm{H}_{2} \mathrm{O}$ & $\mathrm{Cl}$ & $\mathrm{Na}$ & $\mathrm{CHO}$ & Vol. & $\mathrm{Cl}$ & $\mathrm{Na}$ & $\mathbf{K}$ & $\mathrm{N}$ & Glucose \\
\hline L. B. & \begin{tabular}{|c}
\multicolumn{1}{c}{ hours } \\
$0-0.8$ \\
$0.8-5.5$ \\
$5.5-18.3$ \\
$18.3-19.5$
\end{tabular} & $\begin{array}{r}\text { units } \\
30 \\
30 \\
20 \\
0\end{array}$ & $\begin{array}{r}m l . \\
500 \\
600 \\
1740 \\
0\end{array}$ & $\begin{array}{c}\text { m.eq. } \\
76.9 \\
92.3 \\
76.9 \\
0\end{array}$ & $\begin{array}{c}\text { m.eq. } \\
76.9 \\
92.3 \\
76.9 \\
0\end{array}$ & $\begin{array}{r}g m s . \\
0 \\
50 \\
140 \\
0\end{array}$ & $\begin{array}{r}m l . \\
75 \\
194 \\
1010 \\
70\end{array}$ & $\begin{array}{r}m . e q . \\
0.5 \\
13.0 \\
102.9 \\
12.1\end{array}$ & $\begin{array}{r}\text { m.eq. } \\
5.8 \\
10.7 \\
76.1 \\
3.9\end{array}$ & $\begin{array}{r}m . e q . \\
5.7 \\
11.3 \\
20.6 \\
1.4\end{array}$ & $\begin{array}{r}\text { gms. } \\
0.6 \\
2.1 \\
4.2 \\
0.3\end{array}$ & $\begin{array}{c}\text { gms. } \\
0.4 \\
0.9 \\
5.9 \\
0\end{array}$ \\
\hline D. C. (a) & $\begin{array}{r}0 \\
0-5.0 \\
5.0-10.0 \\
10.0-22.8\end{array}$ & $\begin{array}{r}\overline{170} \\
20 \\
55\end{array}$ & $\begin{array}{l}\overline{3011} \\
1000 \\
2865\end{array}$ & $\begin{array}{l}\overline{544.5} \\
153.8 \\
321.3\end{array}$ & $\begin{array}{l}-\overline{544.5} \\
153.8 \\
321.3\end{array}$ & $\begin{array}{l}- \\
22 \\
25 \\
80\end{array}$ & $\begin{array}{r}940 \\
910 \\
680 \\
2040\end{array}$ & $\begin{array}{r}28.2 \\
30.3 \\
54.7 \\
270.0\end{array}$ & $\begin{array}{r}33.8 \\
29.5 \\
45.2 \\
200.5\end{array}$ & $\begin{array}{l}32.3 \\
24.4 \\
19.8 \\
61.3\end{array}$ & $\begin{array}{l}2.3 \\
2.0 \\
2.2 \\
9.9\end{array}$ & $\begin{array}{l}31.1 \\
22.0 \\
12.8 \\
34.8\end{array}$ \\
\hline D. C. (b) & $\begin{array}{r}0 \\
0-5.5 \\
5.5-19.2\end{array}$ & $\begin{array}{l}\overline{120} \\
110\end{array}$ & $\begin{array}{l}- \\
2850 \\
4075\end{array}$ & $\begin{array}{r}-\overline{00.0} \\
572.6\end{array}$ & $\begin{array}{r}-\overline{400.0} \\
572.6\end{array}$ & $\begin{array}{r}\overline{13} \\
263\end{array}$ & $\begin{array}{r}190 \\
1190 \\
3060\end{array}$ & $\begin{array}{r}1.2 \\
47.7 \\
25.6\end{array}$ & $\begin{array}{r}4.4 \\
44.9 \\
119.1\end{array}$ & $\begin{array}{r}5.3 \\
39.0 \\
85.3\end{array}$ & $\begin{array}{l}0.5 \\
4.2 \\
1.2\end{array}$ & $\begin{array}{r}7.2 \\
31.2 \\
98.2\end{array}$ \\
\hline L. D. & $\begin{array}{r}0-5.0 \\
5.0-15.0 \\
15.0-23.0\end{array}$ & $\begin{array}{r}200 \\
70 \\
10\end{array}$ & $\begin{array}{r}3300 \\
2460 \\
810\end{array}$ & $\begin{array}{r}507.6 \\
174.6 \\
92.3\end{array}$ & $\begin{array}{r}507.6 \\
174.6 \\
92.3\end{array}$ & $\begin{array}{r}0 \\
138 \\
5\end{array}$ & $\begin{array}{l}2280 \\
1020 \dagger \\
1030\end{array}$ & $\begin{array}{l}205.8 \\
153.8 \\
206.8\end{array}$ & $\begin{array}{l}219.8 \\
120.2 \\
133.4\end{array}$ & $\begin{array}{l}41.0 \\
10.5 \\
13.2\end{array}$ & $\begin{array}{l}3.9 \\
0.9 \\
2.2\end{array}$ & $\begin{array}{r}51.6 \\
8.2 \\
7.5\end{array}$ \\
\hline R. J.* & $\begin{array}{r}0-4.0 \\
4.0-6.8 \\
6.8-19.2\end{array}$ & $\begin{array}{l}200 \\
100 \\
200\end{array}$ & $\begin{array}{r}1750 \\
850 \\
4150\end{array}$ & $\begin{array}{l}255.8 \\
117.3 \\
625.0\end{array}$ & $\begin{array}{l}264.8 \\
126.3 \\
633.5\end{array}$ & $\begin{array}{r}0 \\
0 \\
116\end{array}$ & $\begin{array}{r}390 \\
80 \\
1305\end{array}$ & $\begin{array}{r}2.7 \\
0.6 \\
136.1\end{array}$ & $\begin{array}{r}2.6 \\
0.3 \\
103.0\end{array}$ & $\begin{array}{r}4.3 \\
1.1 \\
22.5\end{array}$ & $\begin{array}{l}1.4 \\
0.6 \\
8.0\end{array}$ & $\begin{array}{r}19.5 \\
3.2 \\
15.9\end{array}$ \\
\hline J. K. (a) & $0-20.0$ & 240 & 5240 & 769.2 & 769.2 & 132 & 3490 & 256.6 & 264.9 & 74.0 & 8.6 & 155.1 \\
\hline J.K. (b) & $\begin{array}{r}0-8.8 \\
8.8-20.3\end{array}$ & $\begin{array}{r}195 \\
0\end{array}$ & $\begin{array}{l}4050 \\
1500\end{array}$ & $\begin{array}{c}593.0 \\
0\end{array}$ & $\begin{array}{c}593.0 \\
0\end{array}$ & $\begin{array}{r}135 \\
84\end{array}$ & $\begin{array}{r}2580 \\
800\end{array}$ & $\begin{array}{l}145.5 \\
105.0\end{array}$ & $\begin{array}{r}162.1 \\
46.0\end{array}$ & $\begin{array}{l}66.0 \\
10.9\end{array}$ & $\begin{array}{l}5.0 \\
2.9\end{array}$ & $\begin{array}{r}55.2 \\
0.3\end{array}$ \\
\hline W.S. & $\begin{array}{r}0 \\
0-12.2\end{array}$ & $\overline{154}$ & $\overline{4000}$ & $6 \overline{15.3}$ & $\overline{615.3}$ & $\overline{75}$ & $\begin{array}{r}60 \\
1100\end{array}$ & $\begin{array}{r}7.2 \\
164.6\end{array}$ & $\begin{array}{r}5.2 \\
115.9\end{array}$ & $\begin{array}{r}2.2 \\
52.7\end{array}$ & $\overline{6.1}$ & $\overline{26.4}$ \\
\hline M. S.* & $0-17.0$ & 170 & 6255 & 726.3 & 725.7 & 132 & 1663 & 78.5 & 38.1 & 54.9 & 10.9 & 24.3 \\
\hline T. W.* & $\begin{array}{r}0-9.0 \\
9.0-21.0 \\
21.0-25.3\end{array}$ & $\begin{array}{r}330 \\
320 \\
0\end{array}$ & $\begin{array}{l}5000 \\
6760 \\
1575\end{array}$ & $\begin{array}{l}769.1 \\
799.9 \\
153.8\end{array}$ & $\begin{array}{l}769.1 \\
799.1 \\
153.8\end{array}$ & $\begin{array}{r}50 \\
200 \\
50\end{array}$ & $\begin{array}{r}295 \\
1160 \\
220\end{array}$ & $\begin{array}{r}9.1 \\
138.8 \\
30.1\end{array}$ & $\begin{array}{r}5.3 \\
72.9 \\
13.5\end{array}$ & $\begin{array}{r}10.3 \\
20.7 \\
6.2\end{array}$ & $\begin{array}{l}1.1 \\
5.1 \\
1.4\end{array}$ & $\begin{array}{l}4.3 \\
6.7 \\
0.2\end{array}$ \\
\hline J. Ko.* & $\begin{array}{r}0-6.5 \\
6.5-23.0 \\
23.0-71.0\end{array}$ & $\begin{array}{r}120 \\
100 \\
65\end{array}$ & $\begin{array}{l}2000 \\
4000 \\
5285\end{array}$ & $\begin{array}{l}303.4 \\
581.1 \\
588.8\end{array}$ & $\begin{array}{l}303.4 \\
581.1 \\
587.5\end{array}$ & $\begin{array}{r}25 \\
125 \\
221\end{array}$ & $\frac{900}{4750 \dagger}$ & $\frac{113.8}{726.6}$ & $\frac{99.0}{248.9}$ & $\frac{22.9}{36.1}$ & $\frac{2.7}{7.0}$ & $\frac{20.9}{22.4}$ \\
\hline
\end{tabular}

${ }^{*}$ R. J. received 1 m.eq. of $\mathrm{K}$ and 2.8 gms. of $\mathrm{N}$ during each of the three periods. M. S. took in $7.5 \mathrm{~m}$. eq. of $\mathrm{K}$ and $0.55 \mathrm{gm}$. of $\mathrm{N}$ during the 17 hours; $\mathrm{T}$. W. was given $48.7 \mathrm{~m}$.eq. of $\mathrm{K}$ during the second period; J. Ko. took 11.5 m.eq. of K, $1.36 \mathrm{gms}$. of $\mathrm{N}$ in milk and orange juice in addition to $160 \mathrm{cc}$. of ginger ale and $50 \mathrm{cc}$. of grape juice during the 23.0 to 71.0 hour interval. Electrolyte content of the last two items is not known.

† Patient L. D. had emesis of 240 cc., and J. Ko. emesis of 260 cc. which were not analyzed. 
TABLE III

Balances of electrolytes, nitrogen, and carbohydrate*

\begin{tabular}{|c|c|c|c|c|c|c|c|c|c|c|c|c|}
\hline \multirow{2}{*}{ Pt. } & \multirow{2}{*}{$\begin{array}{l}\text { Time } \\
\text { from } \\
\text { start }\end{array}$} & \multicolumn{5}{|c|}{ External balance } & \multicolumn{3}{|c|}{ Extracellular balance } & \multicolumn{3}{|c|}{ Intracellular balancet } \\
\hline & & $\mathrm{Cl}$ & $\mathrm{Na}$ & $\mathrm{K}$ & $\mathrm{N}$ & $\mathrm{CHO}$ & Fluid & $\mathrm{Na}$ & $\mathrm{K}$ & $\mathrm{N}$ & $\mathrm{Na}$ & K \\
\hline L. B. & \begin{tabular}{|c|}
\multicolumn{1}{c|}{ hours } \\
$0-5.5$ \\
$5.5-18.3$ \\
$18.3-19.5$
\end{tabular} & $\begin{array}{l}\text { m.eq. } \\
+151 \\
-\quad 29 \\
-15\end{array}$ & $\begin{array}{l}\text { m.eq. } \\
+147 \\
-\quad 3 \\
-\quad 7\end{array}$ & $\begin{array}{l}\text { m.eq. } \\
-17 \\
-21 \\
-\quad 2\end{array}$ & $\begin{array}{c}\text { gms. } \\
-\quad 2.7 \\
-\quad 4.3 \\
-0.4\end{array}$ & $\begin{array}{r}\text { gms. } \\
+\quad 49 \\
+134 \\
0\end{array}$ & $\begin{array}{l}\text { liters } \\
+1.0 \\
+0.1 \\
-0.3\end{array}$ & $\begin{array}{l}\text { m.eq. } \\
+159 \\
+\quad 71 \\
-\quad 27\end{array}$ & $\begin{array}{r}\text { m.eq. } \\
+3 \\
0 \\
-1\end{array}$ & $\begin{array}{l}\text { gms. } \\
-2.7 \\
-3.8 \\
-0.3\end{array}$ & $\begin{array}{l}m . e q . \\
-\quad 12 \\
-74 \\
+\quad 20\end{array}$ & $\begin{array}{r}\text { m.eq. } \\
-\quad 14 \\
-\quad 12 \\
0\end{array}$ \\
\hline D. C. (a) & $\begin{array}{r}0-5.0 \\
5.0-10.0 \\
10.0-22.8\end{array}$ & $\begin{array}{l}+510 \\
+96 \\
+49\end{array}$ & $\begin{array}{l}+509 \\
+105 \\
+117\end{array}$ & $\begin{array}{l}-25 \\
-20 \\
-61\end{array}$ & $\begin{array}{r}-2.0 \\
-2.3 \\
-9.9\end{array}$ & $\begin{array}{r}0 \\
+\quad 12 \\
+\quad 45\end{array}$ & $\begin{array}{l}+3.9 \\
-0.4 \\
+0.5\end{array}$ & $\begin{array}{l}+561 \\
+91 \\
+197\end{array}$ & $\begin{array}{l}+8 \\
-22 \\
-5\end{array}$ & $\begin{array}{l}+0.6 \\
+3.3 \\
-5.4\end{array}$ & $\begin{array}{r}-53 \\
+\quad 14 \\
-81\end{array}$ & $\begin{array}{l}-\quad 34 \\
-\quad 6 \\
-\quad 43\end{array}$ \\
\hline D. C. (b) & $\begin{array}{r}0-5.5 \\
5.5-19.2\end{array}$ & $\begin{array}{l}+347 \\
+544\end{array}$ & $\begin{array}{l}+349 \\
+450\end{array}$ & $\begin{array}{l}-39 \\
-85\end{array}$ & $\begin{array}{l}-4.2 \\
-\quad 1.2\end{array}$ & $\begin{array}{r}-19 \\
+165\end{array}$ & $\begin{array}{l}+1.5 \\
+3.8\end{array}$ & $\begin{array}{l}+302 \\
+606\end{array}$ & $\begin{array}{l}-8 \\
-2\end{array}$ & $\begin{array}{l}+0.3 \\
+6.9\end{array}$ & $\begin{array}{l}+46 \\
-156\end{array}$ & $\begin{array}{l}-32 \\
-100\end{array}$ \\
\hline L. D. & $\begin{array}{r}0-5.0 \\
5.0-15.0 \\
15.0-23.0\end{array}$ & $\begin{array}{l}+297 \\
+\quad 15 \\
-117\end{array}$ & $\begin{array}{l}+281 \\
+47 \\
-45\end{array}$ & $\begin{array}{l}-41 \\
-11 \\
-13\end{array}$ & $\begin{array}{r}-3.9 \\
-\quad 1.0 \\
-\quad 2.3\end{array}$ & $\begin{array}{r}52 \\
+130 \\
-\quad 3\end{array}$ & $\begin{array}{l}+1.7 \\
+0.1 \\
-0.8\end{array}$ & $\begin{array}{l}+369 \\
-\quad 67 \\
-\quad 57\end{array}$ & $\begin{array}{l}-4 \\
-6 \\
-2\end{array}$ & $\begin{array}{l}-1.9 \\
-0.4 \\
-2.5\end{array}$ & $\begin{array}{l}-88 \\
+115 \\
+\quad 13\end{array}$ & $\begin{array}{l}-32 \\
-\quad 4 \\
-\quad 6\end{array}$ \\
\hline R. J. & $\begin{array}{r}0-4.0 \\
4.0-6.8 \\
6.8-19.2\end{array}$ & $\begin{array}{l}+248 \\
+114 \\
+479\end{array}$ & $\begin{array}{l}+255 \\
+122 \\
+518\end{array}$ & $\begin{array}{r}-4 \\
0 \\
-22\end{array}$ & $\begin{array}{r}+1.3 \\
+\quad 2.2 \\
-\quad 5.3\end{array}$ & $\begin{array}{l}-20 \\
-\quad 3 \\
+100\end{array}$ & $\begin{array}{l}+1.3 \\
+0.4 \\
+2.4\end{array}$ & $\begin{array}{l}+260 \\
+87 \\
+413\end{array}$ & $\begin{array}{l}+1 \\
+1 \\
-4\end{array}$ & $\begin{array}{l}+2.0 \\
+1.0 \\
+0.6\end{array}$ & $\begin{array}{l}-\quad 5 \\
+35 \\
+106\end{array}$ & $\begin{array}{l}-10 \\
-\quad 4 \\
-\quad 19\end{array}$ \\
\hline J. K. (a) & $0-20.0$ & +505 & +494 & -74 & -8.6 & -23 & +4.4 & +647 & +19 & -5.0 & -153 & -81 \\
\hline J. K. (b) & $\begin{array}{r}0-8.8 \\
8.8-20.3\end{array}$ & $\begin{array}{l}+442 \\
-108\end{array}$ & $\begin{array}{l}+425 \\
-49\end{array}$ & $\begin{array}{l}-66 \\
-11\end{array}$ & $\begin{array}{l}-5.0 \\
-2.9\end{array}$ & $\begin{array}{l}+80 \\
+84\end{array}$ & $\begin{array}{l}+2.4 \\
-0.1\end{array}$ & $\begin{array}{l}+507 \\
-136\end{array}$ & $\begin{array}{l}+3 \\
-1\end{array}$ & $\begin{array}{l}-3.3 \\
-1.9\end{array}$ & $\begin{array}{l}-83 \\
+87\end{array}$ & $\begin{array}{l}-62 \\
-\quad 5\end{array}$ \\
\hline W.S. & $0-12.2$ & +443 & +489 & -53 & -6.2 & +49 & +2.7 & +489 & +2 & -1.9 & 0 & -51 \\
\hline M. S. & $0-17.0$ & +638 & +674 & -48 & -10.4 & +108 & +3.8 & +677 & +18 & -2.6 & -3 & -60 \\
\hline T. W. & $\begin{array}{r}0-9.0 \\
9.0-21.0 \\
21.0-25.3\end{array}$ & $\begin{array}{l}+755 \\
+658 \\
+121 \\
\end{array}$ & $\begin{array}{l}+757 \\
+723 \\
+136\end{array}$ & $\begin{array}{r}-11 \\
+28 \\
-\quad 6\end{array}$ & $\begin{array}{l}-1.1 \\
-\quad 5.1 \\
-\quad 1.4\end{array}$ & $\begin{array}{l}+46 \\
+193 \\
+\quad 50\end{array}$ & $\begin{array}{l}+5.4 \\
+3.6 \\
+0.5\end{array}$ & $\begin{array}{l}+768 \\
+697 \\
+103\end{array}$ & $\begin{array}{r}+19 \\
0 \\
-4\end{array}$ & $\begin{array}{l}+1.0 \\
-3.0 \\
+1.5\end{array}$ & $\begin{array}{l}-12 \\
+\quad 25 \\
+\quad 34\end{array}$ & $\begin{array}{r}-31 \\
+\quad 35 \\
-\quad 6\end{array}$ \\
\hline J. Ko. & $\begin{array}{r}0-6.5 \\
23.0-71.0\end{array}$ & $\begin{array}{l}+184 \\
-140\end{array}$ & $\begin{array}{l}+198 \\
+335\end{array}$ & $\begin{array}{l}-23 \\
-25\end{array}$ & $\begin{array}{l}-2.7 \\
-5.6\end{array}$ & $\begin{array}{l}+\quad 4 \\
+199\end{array}$ & $\begin{array}{l}+1.0 \\
-0.6\end{array}$ & $\begin{array}{l}+115 \\
-88\end{array}$ & $\begin{array}{l}-6 \\
-2\end{array}$ & $\begin{array}{l}-1.0 \\
-5.6\end{array}$ & $\begin{array}{l}+84 \\
+423\end{array}$ & $\begin{array}{l}-15 \\
-10\end{array}$ \\
\hline
\end{tabular}

* Balance data are expressed per individual period rather than cumulatively, and are corrected for the electrolytes and $\mathrm{N}$ withdrawn in serum samples.

† Changes in NPN have been taken into account in calculating the balance of cell $\mathrm{N}$; the $\mathrm{K}$ balances represent changes in excess of the transfers associated with the anabolism or catabolism of protein.

$\ddagger 6.5$ to 23.0 hour urine specimen lost and hence calculation of balances for this period is not possible.

of cells throughout these carlicr hours of therapy. It is germane to the argument of this paper to declare at this point that these continued negative balances of cell potassium, even when corrections for protein metabolism have been made, represent an observed fact. Their validity cannot be denied, but rather must be reconciled with the finding that, during these same periods of study, utilization of carbohydrate or its deposition as glycogen was taking place. This interpretation is supported by the finding that the blood sugar concentrations fell progressively while carbohydrate was administered (Table I). The balance data, Tables II and III, further indicate that administered carbohydrate was, in fact, catabolized or stored.
During these same intervals of treatment the patients received parenteral fluids in the amounts indicated in Table II. In the majority of these periods, actually 16 out of 24 , this resulted in a measurable increase in the extracellular volume. These changes, based upon the balance of chloride corrected for alterations in the concentration of the chloride ion in extracellular fluid, are readily identifiable in the second section of Table III as instances in which the extracellular balance of water had risen by 0.5 liter or more. This value has been taken as a reasonable approximation of a change which definitely falls outside the range of the errors inherent in these methods of analyses and calculations $(10,11,14)$. It is to be noted that in the remaining eight of the 24 periods the 
extracellular space either failed to change significantly, or actually declined in volume. These findings clearly suggest that in many, but not all, of our patients the extracellular fluid was diluted by the administration of potassium-free solutions.

\section{DISCUSSION}

The data presented have established the following facts: (1) patients undergoing therapy for diabetic acidosis continue to excrete measurable amounts of potassium in the urine, (2) during this interval, if no potassium is administered, the over-all balance of cell potassium remains consistently negative, even though potassium is at times deposited with protein and the carbohydrate balances become positive, and (3) fluid therapy with potassium-free solutions during this time frequently results in an expansion of the extracellular volume.

In view of the above statements it is reasonable to suggest that in any particular patient recovering from diabetic acidosis, continued urinary loss of potassium, as long as potassium-containing urine is elaborated, will be a contributory factor in the development of hypopotassemia. Similarly, re-expansion of the body fluids, particularly of the extracellular volume, by means of solutions devoid of potassium will also tend to produce low serum and interstitial fluid concentrations of potassium. Finally, the deposition of potassium with glycogen or with protein will remove potassium from the extracellular fluid, and thereby cause hypokaliemia $(10,15)$. Obviously, the inportance of any one of these processes in the origin of hypokaliemia will vary with its magnitude and direction. Analyses of our own group of cases, based on estimation of the total amount of potassium in the extracellular compartment at the start and at the end of an interval during which the potassium concentration had dropped, indicate that the dilution of body fluid with potassium-free fluid is at times sufficiently large to account for the entire decrease in serum potassium concentration. Less often the drop appears to be explicable by a combination of dilution of body fluids and continued urinary losses of potassium. Only rarely can the low levels be explained solely by urinary losses of the cation (Table III).
In no instance, irrespective of the roles played by dilution or by renal excretion in the production of hypokaliemia, is the movement of potassium into cells precluded. This is true even though the net balances of cell potassium are negative. As already indicated, nitrogen was deposited with potassium in the cells in a number of the periods. Similarly, glycogen must have been deposited with potassium in the liver during the recovery phase (15). This is apparent from the in vivo studies of others and is supported by the positive carbohydrate balances in our own series (16). It is clear that though these processes undoubtedly occurred, they were usually not, in our series of cases, of sufficient magnitude to cancel entirely the tendency for cells to lose potassium during the early treatment and recovery phases of diabetic acidosis. The explanation for this negative net balance of cell potassium is not available. It may well be merely a continuation, at a reduced pace, of mechanisms which result in cell potassium loss during acidosis.

\section{SUMMARY AND CONCLUSION}

A small series of juvenile and young adult diabetics undergoing therapy for acidosis has been studied by means of the balance method. It appears that during this early phase of therapy dilution of body fluids by potassium-free solutions, continued urinary loss of potassium, and migration of potassium out of the extracellular phase into cells with the deposition of protein or of glycogen, even though the over-all balances of cell potassium remain negative, may each contribute to the hypokaliemia which develops.

\section{BIBLIOGRAPHY}

1. Holler, J. W., Potassium deficiency occurring during the treatment of diabetic acidosis. J. A. M. A., 1946, 131, 1186.

2. Nicholson, W. M., and Branning, W. S., Potassium deficiency in diabetic acidosis. J. A. M. A., 1947, 134, 1292.

3. Martin, H. E., and Wertman, M., Serum potassium, magnesium, and calcium levels in diabetic acidosis. J. Clin. Invest., 1947, 26, 217.

4. Guest, G. M., and Rapoport, S., Electrolytes of blood plasma and cells in diabetic acidosis and during recovery. Proc. Amer. Diab. A., 1947, 7, 97.

5. Butler, A. M., Talbot, N. B., Burnett, C. H., Stanbury, J. B., and MacLachlan, E. A., Metabolic studies in diabetic coma. Tr. A. Am. Physicians, 1947, 60, 102. 
6. Frenkel, M., Groen, J., and Willebrands, A. F., Reduction of serum potassium content with manifestations of generalized muscular weakness and a cardiovascular syndrome during treatment of diabetic coma. Nederl. tijdschr. v. geneesk., 1947, 91, 1704. (Abstracted in J. A. M. A., 1947, 135, 602.)

7. Tuynman, P. E., and Wilhelm, S. K., Potassium deficiency associated with diabetic acidosis. Ann. Int. Med., 1948, 29, 356.

8. Logsdon, C. S., and McGavack, T. H., Death, probably due to potassium deficiency, following control of diabetic coma. J. Clin. Endocrinol., 1948, 8, 658.

9. Danowski, T. S., Peters, J. H., Rathbun, J. C., Quashnock, J. M., and Greenman, L., Studies in diabetic acidosis and coma, with particular emphasis on the retention of administered potassium. J. Clin. Invest., 1949, 28, 1.

10. Elkinton, J. R., and Winkler, A. W., Transfers of intracellular potassium in experimental dehydration. J. Clin. Invest., 1944, 23, 93.
11. Elkinton, J. R., Winkler, A. W., and Danowski, T. S., Transfers of cell sodium and potassium in experimental and clinical conditions. J. Clin. Invest., 1948, 27, 74.

12. Darrow, D. C., The retention of electrolyte during recovery from severe dehydration due to diarrhea. J. Pediat., 1946, 28, 515.

13. Atchley, D. W., Loeb, R. F., Richards, D. W., Jr., - Benedict, E. M., and Driscoll, M. E., On diabetic acidosis; detailed study of electrolyte balances following the withdrawal and reestablishment of insulin therapy. J. Clin. Invest., 1933, 12, 297.

14. Elkinton, J. R., Winkler, A. W., and Danowski, T. S., Inactive cell base and the measurement of changes in cell water. Yale J. Biol. \& Med., 1944, 17, 383.

15. Fenn, W. O., The deposition of potassium and phosphate with glycogen in rat livers. J. Biol. Chem., 1939, 128, 297.

16. Bondy, P. K., Studies of carbohydrate metabolism in normal and diabetic patients by the liver catheterization technic. J. Clin. Invest., 1948, 27, 526. 